Gynäkologische Endokrinologie 2018 · 16:1-2 https://doi.org/10.1007/s10304-017-0168-5 Online publiziert: 22. November 2017 (c) Springer Medizin Verlag GmbH, ein Teil von Springer Nature 2017

CrossMark

\author{
H. Kentenich $\cdot$ M. David ${ }^{2} \cdot$ W. Küpker ${ }^{3}$ \\ ${ }^{1}$ Fertility Center Berlin, Berlin, Deutschland \\ ${ }^{2}$ Klinik für Gynäkologie, Charité - Universitätsmedizin Berlin, Campus Virchow-Klinikum, Berlin, \\ Deutschland \\ ${ }^{3}$ Abteilung für Gynäkologische Endokrinologie, Endometriose und Reproduktionsmedizin, Frauenklinik, \\ Baden-Baden/Bühl, Standort Bühl, Klinikum Mittelbaden Bühl, Bühl, Deutschland
}

\title{
Soziokulturelle Aspekte und Frauengesundheit
}

die damit verbundenen Besonderheiten und Probleme Klinikwirklichkeit waren.

Die gesundheitliche Versorgung von Migrantinnen und Migranten stellt ein Gesundheitssystem meist vor besondere Herausforderungen. Kulturelle, sprachliche und andere Kommunikationsbarrieren können den Aufbau einer tragfähigen Arzt-Patientin-Beziehung, eine gute Verständigung und das erfolgreiche Erfassen des eigentlichen Anliegens des Patienten erschweren. Interkulturelles Wissen und interkulturelle Kompetenz, Empathie und das Hinterfragen eigener Positionen sind für die Bewältigung solcher Situationen erforderlich.

Der Beitrag von Theda Borde fokussiert auf die Themen Kommunikation und Sprache. Etwa $20 \%$ der in Deutschland lebenden Bevölkerung haben aktuell einen sog. Migrationshintergrund. Bei einem Teil dieser Menschen muss man von Problemen im Bereich Sprache und Kommunikation ausgehen. Kommunikationsprobleme können beispielsweise $\mathrm{zu}$ unterschiedlichen Einschätzungen der Krankheitsschwere und der Dringlichkeit der Therapie führen. Die häufig im Praxis- und Klinikalltag praktizierte Vorgehensweise, Angehörige als „Adhoc-Dolmetscher“ zu nutzen, stellt zwar eine einfache Problemlösung dar, führt aber, wie Theda Borde in ihrem Beitrag u. a. ausführt, zum Verzerren, Filtern und Verschweigen von Informationen. Der Aufbau eines flächendeckenden Netzes von geschulten Dolmetschern/ professionellen Sprachmittlern in der Bundesrepublik Deutschland, die durch die Kliniken und Praxen kostenfrei ge- nutzt werden können, erscheint daher sinnvoll, ja dringend geboten.

Wenn Migrantinnen eine Arztpraxis oder ein Krankenhaus aufsuchen, dann ist neben medizinischer auch interkulturelle Kompetenz gefragt, denn sowohl die Medizin selbst als auch Krankheiten sind in ein kulturelles System eingebunden. Krankheits- und Behandlungsvorstellungen von Migranten sind u. U. ganz andere, als die des behandelnden, durch das naturwissenschaftlich ausgerichtete Studium und die europäische Sichtund Denkweise geprägten behandelnden Arztes. In seinem Artikel geht Matthias David daher auf die Unterschiede in der sog. subjektiven Krankheitstheorie und mögliche kulturelle Unterschiede von Krankheitssymptomen am Beispiel einiger gynäkologischer Krankheitsbilder ein. Beeindruckend ist beispielsweise die häufigere Hyperemesis gravidarum bei Migrantinnen - ein Krankheitsbild, bei dessen Therapie gleichermaßen medizinische (somatische Verursachung der Übelkeit) und psychische Aspekte (ambivalente Gefühle im Zusammenhang mit der Schwangerschaft) einbezogen werden müssen.

Hartmut Kress fokussiert auf die Reproduktionsmedizin und die religiösen und ethischen Vorbehalte. Bekannt sind die ablehnende Haltung der römisch-katholischen Kirche und die zurückhaltende Haltung der evangelischen Kirche. Da bei den Patientinnen und Paaren die religiöse Gebundenheit sehr unterschiedlich ist, hat das nur geringe Auswirkungen auf die Behandlungshäufigkeit. Wissenswert ist die Haltung im Judentum und Islam zu Fragen der Reproduktion, wobei inner- 
halb des Islam die schiitische und die sunnitische Herangehensweise unterschiedlich sind. In der alltäglichen Medizin sollte dies dazu führen, dass der religiöse und ethische Hintergrund der Patientinnen und Paare ein wichtiger Bestandteil in der Entscheidungsfindung sind.

Herta Seyler geht sehr detailliert auf die gesundheitliche Versorgung von Flüchtlingen auf der Grundlage des Asylbewerberleistungsgesetzes ein. Wichtig für die Praxis ist der Hinweis, dass diejenigen Patientinnen und Patienten, die geduldet oder bereits 15 Monate im Asylverfahren sind, Leistungen nach regulärer gesetzlicher Krankenversicherung (GKV) erhalten können. Alle Flüchtlinge haben in Deutschland nach einer entsprechenden Registrierung das Recht, in medizinischen Notfällen behandelt zu werden. Im Zweifel hilft eine Anfrage bei den Erstaufnahmeeinrichtungen weiter. Der Hinweis auf Netzwerke für Nichtversicherte (Büros für medizinische Flüchtlingshilfe) erscheint sinnvoll. Diese Büros finden sich in vielen Großstädten. Die nächsten Jahre werden sicher weiterhin davon geprägt sein, dass Flüchtlinge in unterschiedlichem Ausmaß das deutsche Gesundheitssystem in Anspruch nehmen.

„Verstehen und verstanden werden - das ist Heimat."

(Bundespräsident Frank-Walter Steinmeier zum Tag der Deutschen Einheit, 03.10.2017)

\section{Lo thasuic}

H. Kentenich

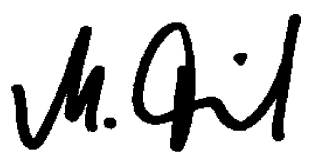

M. David
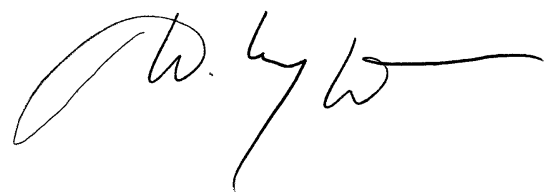

W. Küpker

\section{Korrespondenzadresse}

Prof. Dr. H. Kentenich

Fertility Center Berlin

Spandauer Damm 130, 14050 Berlin,

Deutschland

kentenich@fertilitycenterberlin.de

Interessenkonflikt. H. Kentenich hält Vorträge auf Veranstaltungen von Merck Serono, Dr. Kade und ReproFacts. M. David und W. Küpker geben an, dass kein Interessenkonflikt besteht.

\section{Stillen und Säuglings- ernährung in Deutschland}

Daten für die praxisnahe Beratung junger Familien

Mit der Ernährung in der frühen Kindheit werden Weichen für die Gesundheitsförderung und Prävention weitverbreiteter Krankheiten im Erwachsenenalter gestellt. Zwar gibt es in Deutschland wissenschaftlich basierte Empfehlungen für die Ernährung von Säuglingen, es fehlen aber Daten, ob und wie gut diese Empfehlungen in der Praxis umgesetzt werden.

Hier setzt die neue, groß angelegte Studie zum Stillen und zur Säuglingsernährung SuSe II an. Sie ist eine Fortschreibung der vor 20 Jahren am damaligen Forschungsinstitut für Kinderernährung Dortmund (FKE) durchgeführten SuSe Studie. Die neue Studie untersucht, wie sich das Stillverhalten und die Ernährung von Säuglingen im 1. Lebensjahr in Deutschland seither entwickelt haben und welche Faktoren die Einhaltung der Empfehlungen fördern oder aber behindern.

Die Studie ist für 2 Jahre angesetzt. Wie in der Vorgängerversion handelt es sich um eine Querschnittstudie zum Stillmanagement in bundesdeutschen Geburtskliniken und daran anschließend prospektive Follow-up Befragungen bei dort rekrutierten Mutter-Kind-Paaren im 1. Lebensjahr des Kindes.

Die Anwerbung der Mütter in den partizipierenden Kliniken beginnt Anfang 2018. Interessierte Kliniken können sich bis Ende 2017 für die Teilnahme anmelden.

Bei Fragen zur Teilnahme an der Studie wenden Sie sich bitte an: Prof. Dr. Mathilde Kersting Forschungsdepartment Kinderernährung (FKE), Universitätskinderklinik Bochum Alexandrinenstraße 5

44791 Bochum

Fon: +49 234 / 5092615

mathilde.kersting@ruhr-uni-bochum. de

Quelle: Universitätskinderklinik Bochum, Forschungsdepartment Kinderernährung (FKE) 